\title{
OPEN Algal magnetic nickel oxide nanocatalyst in accelerated synthesis of pyridopyrimidine derivatives
}

\begin{abstract}
Javad Moavi ${ }^{1}$, Foad Buazar ${ }^{1 \bowtie}$ \& Mohammad Hosein Sayahi
This research presents a novel biological route for the biosynthesis of nickel oxide nanoparticles (NiO NPs) using marine macroalgae extract as a reducing and coating agent under optimized synthesis conditions. XRD and TEM analyses revealed that phytosynthesized NiO NPs are crystalline in nature with a spherical shape having a mean particle size of $32.64 \mathrm{~nm}$. TGA results indicated the presence of marine-derived organic constituents on the surface of NiO NPs. It is found that biogenic NiO NPs with BET surface area of $45.59 \mathrm{~m}^{2} \mathrm{~g}^{-1}$ is a highly efficient catalyst for benign one-pot preparation of pyridopyrimidine derivatives using aqueous reaction conditions. This environmentally friendly procedure takes considerable advantages of shorter reaction times, excellent product yields (up to $96 \%$ ), magnetically viable nanocatalyst (7 runs), low catalyst loadings, and free toxic chemical reagents.
\end{abstract}

In recent years, the marine realm contribution to the production of versatile nanoparticles has undergone significant growth across international research communities since its economic, rapid, and consequently, environmental procedures render a viable template for the biosynthetic route ${ }^{1,2}$. Marine organisms (e.g. algae, yeast, bacteria, fungal species) owing to possessing a broad variety of electron-rich phytochemicals such as polyphenols, carbohydrates, polyols, alkaloids, proteins, polysaccharides, peptides, and amino acids, establish a sing-step and comprehensive platform for the simultaneous reduction of metal cations and stabilization of biofabricated nanoparticles ${ }^{3}$. In contrast to commonly employed physical and chemical approaches, the sheer growing demand for uses of green processes presumably due to environmental compatibility, simplicity, and the absence of detrimental chemical reagents ${ }^{4}$.

Algae are highly diversified naturally occurring microbes and inhabitants of various aquatic environments such as marine- and fresh-water. These microorganisms are commercially and biologically of great importance since they contain valuable phytoconstituents that address the global market needs through virtual engagement in commercial products, for instance, cosmetics, biofuel, and drugs. In the aspect of biosynthesis, marine algae are known as "bionanofactory", and hence, different nanoscale materials including alumina, zinc oxide, gold, have been synthesized through miscellaneous algae as a competent green synthesis method ${ }^{5-7}$.

Catalyst is a focal point in enormous chemical reactions and has been gaining a surge of popularity particularly in academic and industrial synthetic reactions areas over the past decades. Interestingly, convergence between nanoscience and chemistry led to the advent of nanocatalysts in which as a result of immense surface areas and expansive catalytic capabilities, have attracted great attention of many organic chemists in a myriad of catalyzed-reactions ${ }^{8,9}$. Apart from using a trace amount of catalyst, nanocatalysis processes dramatically enhance the contact between the active component of the catalyst and reactants and therefore increase the rate and yield of chemical reactions ${ }^{10,11}$. A vast majority of the heterogeneous and homogenous catalysts are pertinent to transition metal nanoparticles due to their unprecedented physicochemical properties ${ }^{12-15}$. Among these tremendous efforts, nickel oxide nanoparticles have been developed as an efficient catalyst in the chemical synthesis of a wide range of valuable organic compounds such as spiro and condensed indole derivatives ${ }^{16}$, aromatic heterocycle ${ }^{17}$, 5 -substituted 1 h-tetrazoles ${ }^{18}$, quinolines ${ }^{19}$, spirooxindoles ${ }^{20}$, polyhydroquinolines, and sulfoxidation ${ }^{21}$. There are a variety of green methods that have been addressed in the construction of the nickel oxide nanoparticles

\footnotetext{
${ }^{1}$ Department of Marine Chemistry, Khorramshahr University of Marine Science and Technology, P.O. Box 669, Khorramshahr, Iran. 'Department of Chemistry, Payame Noor University, P.O. Box 19395-3697, Tehran, Iran. ${ }^{\boxplus e m a i l: ~ f b @ k m s u . a c . i r ~}$
} 
<smiles>C=CC(=O)Nc1nc2c(Nc3ccc(F)c(Cl)c3)ncnc2cc1OCCCN1CCOCC1</smiles>

Tyrosine kinase inhibitors<smiles>CC(C)(C)c1nc2[nH]c(=S)[nH]c(=O)c2cc1-c1ccc(Cl)cc1</smiles>

analgesic<smiles>O=c1[nH]c(S[Al])nc2nc(Br)cc([Al])c12</smiles>

anti-inflammatory<smiles>Cn1c(=O)c(-c2c(Cl)cccc2Cl)cc2cnc(Nc3ccccc3)nc21</smiles>

TKI-28

Scheme 1. Selected pyridopyrimidine compounds with pharmaceutical properties ${ }^{27}$.

(NiO NPs) for various application ${ }^{22}$. It is worth mention that the plant-assisted bioreduction strategy for $\mathrm{NiO}$ NPs fabrication has received glob attention as a renewable and sustainable supplier ${ }^{23-25}$.

Multicomponent Reactions (MCRs) are propitious condensation reactions ${ }^{26,27}$, in which integrate moieties of several starting materials to produce a desirable product ${ }^{28,29}$. This efficient chemical synthesis toolbox is of great interest due to high selectivity, bond-construction efficiency, and sustainability in providing complex molecules with promoted diversity ${ }^{12,15,30}$. Moreover, MCR are considered as a combinatorial synthesis approach in which a chemical reaction of all materials is occurred in one pot in a single step fashion producing a wide variety of organic compounds with relatively strict avoidance of waste formation ${ }^{31-34}$.

Pyridopyrimidines and their fused heterocycles derivatives are of paramount importance due to their significant biological and pharmacological activities. Moreover, owing to the involvement of pyridopyrimidine skeleton in some essential medicinal drugs, they hold great promise in the area of pharmaceutical science. Compounds possessing the pyrido[3,2-d]pyrimidines scaffold demonstrate a broad spectrum of biological activities such as antiviral $^{35,36}$, antimicrobial ${ }^{37}$, antihypertensive, anti-tumor ${ }^{38}$, antihistaminic ${ }^{39}$, antimalarial, potent inhibitor of protein kinases $^{40}$, treatment of diarrhea ${ }^{41}$, anti-inflammatory and analgesic activity ${ }^{42}$, along with other medicinal applications (Scheme 1$)^{43}$. Due to their large variety of characteristics, there has been a growing demand in the design and production of pyridopyrimidine nucleus derivatives in particular those derived from proactive biological procedures.

In this study, proceeding our interest toward green nanoparticle synthesis and application ${ }^{44}$, we report a novel biosynthetic method to produce NiO NPs through electron-rich marine algae extract by optimizing these reaction conditions. Afterward, the catalytic efficiency of marine-assisted NiO NPs was explored in the eco-friendly synthesis of pyridopyrimidine derivatives by one-pot three-component elaborated in green conditions. The entire synthetic reaction was performed in water as a highly desirable solvent where increases the environmental impact and economical perspective of the designed protocol ${ }^{45}$. Based on the literature survey, this is the new report on the use of marine algae-assisted $\mathrm{NiO}$ NPs as a heterogenetic catalyst in organic synthesis of a library of pyridopyrimidine heterocyclic compounds through newly four-component condensation.

\section{Results and discussion}

UV-visible spectroscopy analysis. The initial evidence of NiO NPs formation was revealed when the solution color changed from brown to dark green. Correspondingly, the UV-vis spectrum demonstrated a characteristic absorbance peak at $330 \mathrm{~nm}$ indicating biofabrication of NiO NPs in the extract of marine algae biomass (Fig. 1a, b). The completion of the reaction was monitored in UV visible spectra as a function of time (5 min, $10 \mathrm{~min}, 20 \mathrm{~min}, 30 \mathrm{~min}$, and $1 \mathrm{~h}$ ). The results show that the apex of the absorption peak intensity was observed at $30 \mathrm{~min}$ indicating the reduction of nickel ions to zero valance metallic nickel atom. Moreover, no sensible changes have been observed in peak position when the sample stored up to six months in the laboratory, indicating high stability of bioproduced NiO NPs in aqueous green media. Previous studies reported the optical absorption peak of biological NiO NPs in the range of 330-350 nm using green synthesis methods ${ }^{24,25,46}$ which are in good agreement with our results. 


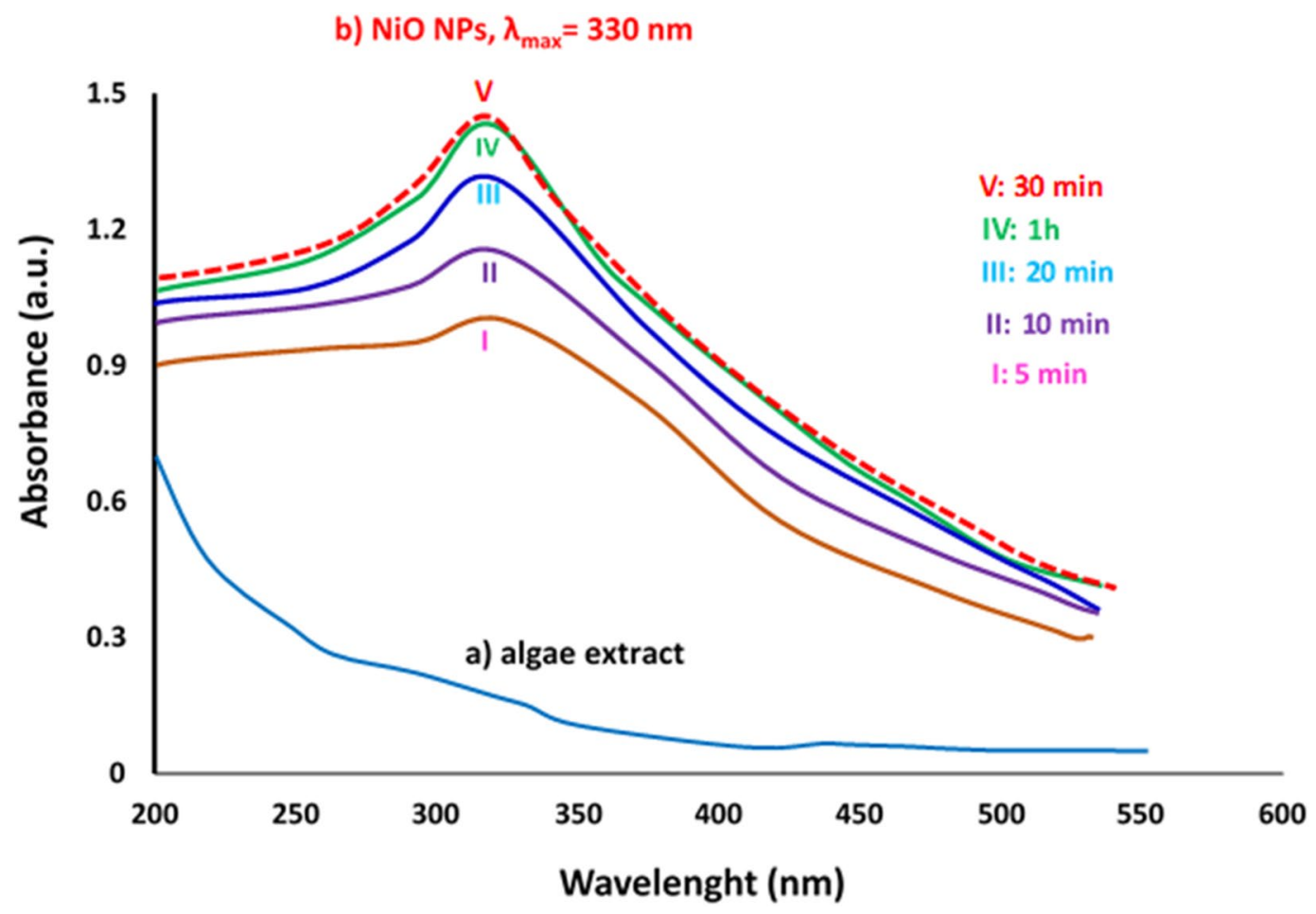

Figure 1. UV-visible spectra of (a) organic seaweed extract, and (b) optimization of biogenic NiO NPs as a function of time.

\begin{tabular}{|c|c|c|c|c|c|c|}
\hline $2 \boldsymbol{\theta},\left(^{\circ}\right)$ & $\begin{array}{c}\text { FWHM, } \\
\left({ }^{\circ}\right)\end{array}$ & $\begin{array}{c}\text { Size, d } \\
(\mathrm{nm})\end{array}$ & $\begin{array}{c}\text { D spacing, } \\
(\mathrm{nm})\end{array}$ & $\mathrm{hkl}$ & $\begin{array}{c}\text { Latticeparameter, } \\
\text { for NiO NPs, }(\AA)\end{array}$ & $\begin{array}{c}\text { Standard lattice } \\
\text { parameter for NiO, }(\AA)\end{array}$ \\
\hline 43.35 & 0.479 & 18.00 & 0.208521 & $(200)$ & $\mathrm{a}=\mathrm{b}=\mathrm{c}=4.170$ & 4.176 \\
\hline
\end{tabular}

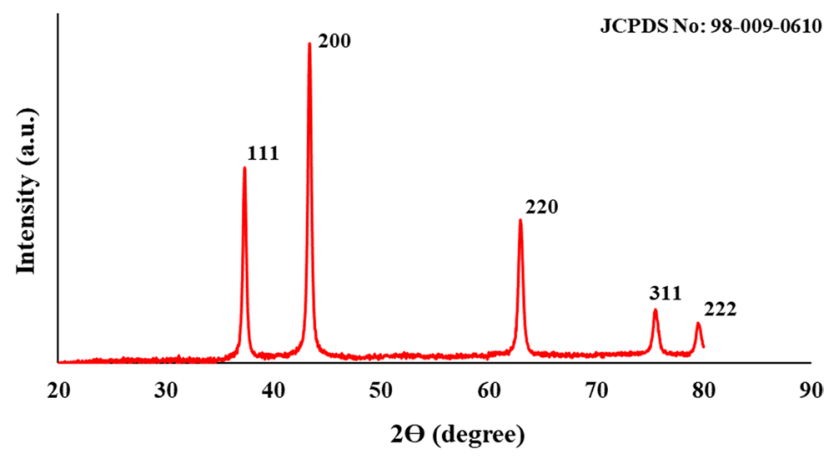

Figure 2. XRD pattern and crystal data of biosynthesized NiO NPs using red marine algae extract.

XRD analysis. The phase structure of marine algae-mediated NiO NPs is further scrutinized by the X-ray diffraction technique. The crystal data $\left(\alpha=\beta=\gamma=90^{\circ}\right.$, lattice parameters, $\left.a=b=c=4.1790 \AA\right)$, prominent diffraction angles and miller indexes (hkl) at $37.45^{\circ}(111), 43.15^{\circ}(200), 62.77^{\circ}(220), 75.34^{\circ}(311)$, and $79.64^{\circ}(222)$, deduced from XRD pattern revealed that biogenic NiO NPs possess face center cubic (FCC) crystalline structure with FM3-M space group (Fig. 2) ${ }^{47}$. Owing to the nonappearance of impurity peaks, the biofabricated nanoparticles were highly pure in nature. In addition, our obtained data are well-matched with JCPDS No: 98-009-0610, showing similar results to literature reports concerning biosynthesized $\mathrm{NiO} \mathrm{NPs}^{48,49}$. Based on the high intensity of Bragg peak at $43.35^{\circ}(200)$, the calculated median crystallite size of NiO NPs was $18 \mathrm{~nm}$ using Debye-Scherrer's formula.

TEM characterization. The particle size and morphology and of NiO NPs were determined through the TEM analysis. TEM images confirmed that the vast majority of the particles were particles relatively non-spherical in shape, however, they appear in uniform and smooth morphology in the green synthesis platform as 

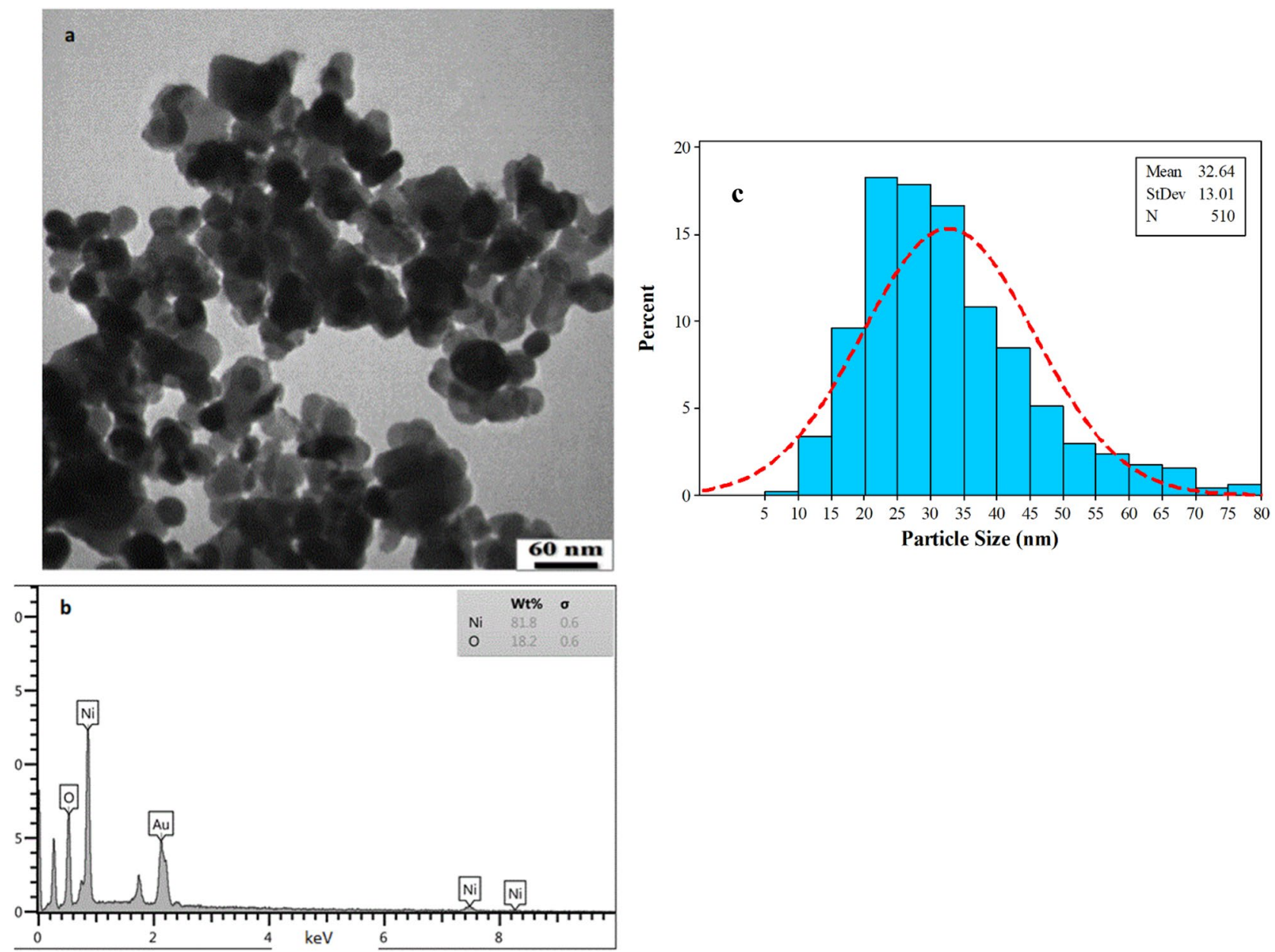

Figure 3. (a) The TEM image at $60.000 \mathrm{KX}$ magnification, (b) EDX analysis, and (c) histogram of particle size distribution of algal NiO NPs.

highlighted in Figure 3a. It can be noted that some regions of the sample appear darker in TEM image of bioprepared NiO NPs perhaps as a result of variation in thickness or high mass density of particles. Furthermore, the histogram of TEM image assay exhibited that biogenic NiO NPs possess a median diameter of $32.64 \mathrm{~nm}$ obtained through counting 510 particles as performed by ImageJ software (Figure 3c) ${ }^{50}$. EDX analysis confirmed the major components of the sample with nickel and oxygen atoms indicating the purity of biogenic Ni NPs (Figure $3 \mathrm{~b}$ ). The Au element detected in the EDX analysis was resulted from the gold-coated grid used for SEM specimen preparation.

FTIR analysis. FTIR spectroscopy is a powerful tool to determine organic constituents qualitatively in seaweeds and plants ${ }^{5}$. The recorded spectrum presents a wide variety of prominent functional groups on the surface of biological NiO NPs as depicted in Fig. 4. The results of fingerprint regions of main functional groups on surface of fresh NiO NPs were also summarized in Table 1. The estimation of the class of organic moieties in the seaweeds is of great value since it would elucidate their role in the reduction and capping of freshly produced nanoparticles in the green medium. The strong wide band at $3411 \mathrm{~cm}^{-1}$ is characteristic of electron-rich $\mathrm{N}-\mathrm{H}$ and $\mathrm{O}-\mathrm{H}$ stretching vibrations, presenting amino acid and hydroxyl groups of polysaccharides. The weak $\mathrm{C}-\mathrm{H}$ stretching mode at $2945 \mathrm{~cm}^{-1}$ is attributed to the $\mathrm{CH}_{2}$ and $\mathrm{CH}_{3}$ groups of aliphatic compounds. The characteristic absorption peak around $1640 \mathrm{~cm}^{-1}$ is on account of the presence of $\mathrm{C}=\mathrm{O}$, pertaining to ester groups. The $\mathrm{C}=\mathrm{C}$ absorption band representative of the lignin is observed at $1545 \mathrm{~cm}^{-1}$. The absorption band centered at $1322 \mathrm{~cm}^{-1}$ indicates $\mathrm{S}=\mathrm{O}$ stretching of sulfated polysaccharide entity. Doublet symmetric $\mathrm{C}-\mathrm{O}$ vibration peaks appeared at 1030 and $1193 \mathrm{~cm}^{-1}$ may belong to ethers and glycosidic of carbohydrates. The advent of strong characteristic vibrations at 525 and $685 \mathrm{~cm}^{-1}$ indicates $\mathrm{Ni}-\mathrm{O}$ bonds in the fingerprint region ${ }^{24,48}$. These results show the interaction of algal electron donor biomolecules with nickel cations maybe lead to the reduction as well as coating as-prepared $\mathrm{NiO}$ NPs. Apparently phytochemical presence in plant or microorganism extracts especially oxygen-contain biomolecules are acted through an oxidation-reduction mechanism, affording an appropriate reaction medium to generate eco-friendly nanomaterials ${ }^{51}$. 




Figure 4. FTIR spectra of marine algae-mediated synthesized NiO NPs.

\begin{tabular}{|l|l|l|l|}
\hline Entry & Functional group & Adsorption $\left(\mathrm{cm}^{-1}\right)$ & References \\
\hline 1 & $\mathrm{O}-\mathrm{H}, \mathrm{N}-\mathrm{H}$ stretch & $3411 \mathrm{~cm}^{-1}$ & 52 \\
\hline 2 & $\mathrm{C}-\mathrm{H}$ stretch & $2945 \mathrm{~cm}^{-1}$ & 53 \\
\hline 3 & $\mathrm{C}=\mathrm{O}$ stretch & $1640 \mathrm{~cm}^{-1}$ & 52 \\
\hline 4 & $\mathrm{C}=\mathrm{C}$ stretch & $1545 \mathrm{~cm}^{-1}$ & 54 \\
\hline 5 & S $=\mathrm{O}$ stretch & $1322 \mathrm{~cm}^{-1}$ & 55 \\
\hline 6 & C $-\mathrm{O}$ stretch & $1193 \mathrm{~cm}^{-1}, 1030 \mathrm{~cm}^{-1}$ & 52 \\
\hline 7 & $\mathrm{Ni}-\mathrm{O}$ stretch & $525 \mathrm{~cm}^{-1}, 685 \mathrm{~cm}^{-1}$ & 24,48 \\
\hline
\end{tabular}

Table 1. FTIR characteristic absorption peaks of the engaged biomolecules in marine-mediated NiO NPs.

TGA investigation. The TGA technique is a powerful tool to explore green-assisted nanoparticles that encompassed organic biomolecules on their surface. The results of the TGA plot were depicted in Fig. 5. The first steady weight loss rate of $14 \%$ was occurred at the range of temperatures between 70 and $120{ }^{\circ} \mathrm{C}$ which is assigned to the vaporized $\mathrm{H}_{2} \mathrm{O}$ molecules absorbed on the surface of as-prepared NiO NPs. The second major loss with a proportional amount of $41 \%$ was observed at the temperature range of $220-420^{\circ} \mathrm{C}$ most probably due to decomposing of marine-derived organic compounds that decorated the NiO NPs. Consequently, TGA curve findings are in good agreement with FTIR results fortifying the presence of propitious biofunctional groups which in turn could enhance the reduction of metallic nickel cations and coat the peripheral surface of freshly bioengineered nanoparticles.

VSM properties of NiO NPs. The vibrating sample magnetometer (VSM) analyzer was used to measure the probable magnetic amount of biologically formed NiO NPs. Figure 6 illustrates the magnetization $\mathrm{M}$ curve of green $\mathrm{NiO} \mathrm{NPs}$, after annealing, at fields of -10 and +10 Oersted. The $\mathrm{M}$ and $\mathrm{H}$ lines are not intersected in the entire curve indicating that coercivity $\left(H_{c}\right)$ and remanent magnetization $\left(M_{r}\right)$ were naught. As a result, algal $\mathrm{NiO} N$ s could deem as superparamagnetic material. The saturation magnetization $\left(\mathrm{M}_{\mathrm{s}}\right)$ is the maximum value of magnetic induction and is size-dependent in which crystal lattice defects, inferior agglomeration state of the particles, size smallness, and higher surface-to-volume ratio would induce higher magnetic properties. Based on the VSM graph, Ms value of biogenic NiO NPs was measured as $0.198 \mathrm{emu} / \mathrm{g}$ which displays superior magneti-

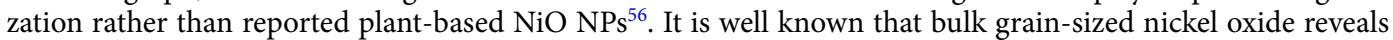
antiferromagnetic susceptibility at room temperature ${ }^{57}$, whereas superparamagnetism is evidently a function of particle size ${ }^{58}$ for nanoparticles particularly below $20 \mathrm{~nm}$ as indicated for green NiO NPs.

BET analysis. The BET curve of bio-assisted NiO NPs can be seen in Fig. 7. According to the result obtained from the BET isotherm measurement, the value of NiO NPs surface area, micropore volume, and average pore 


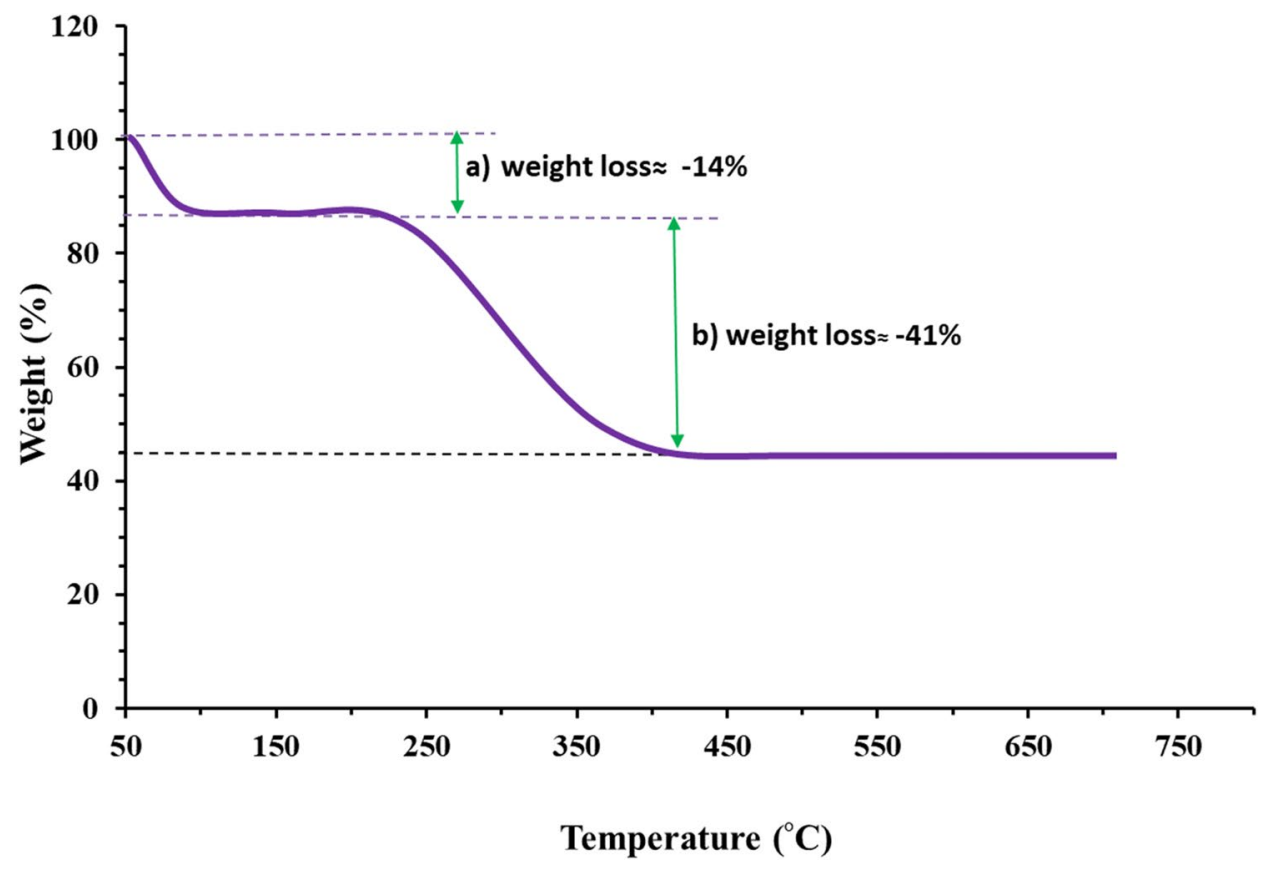

Figure. 5. TGA curve of green NiO NPs using marine red algae extract.

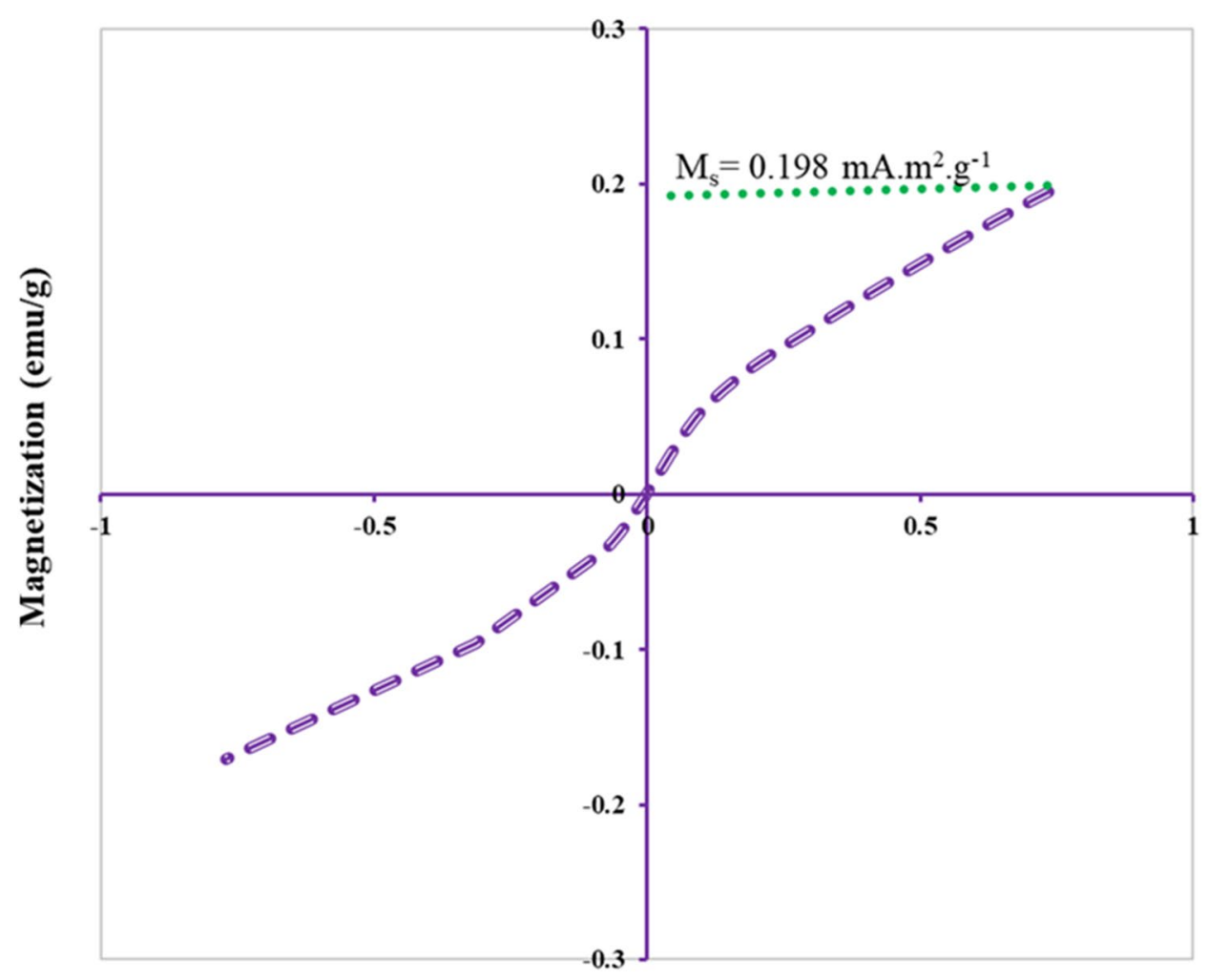

Applied feild (Oe)

Figure 6. VSM magnetization curve of algal NiO NPs. 


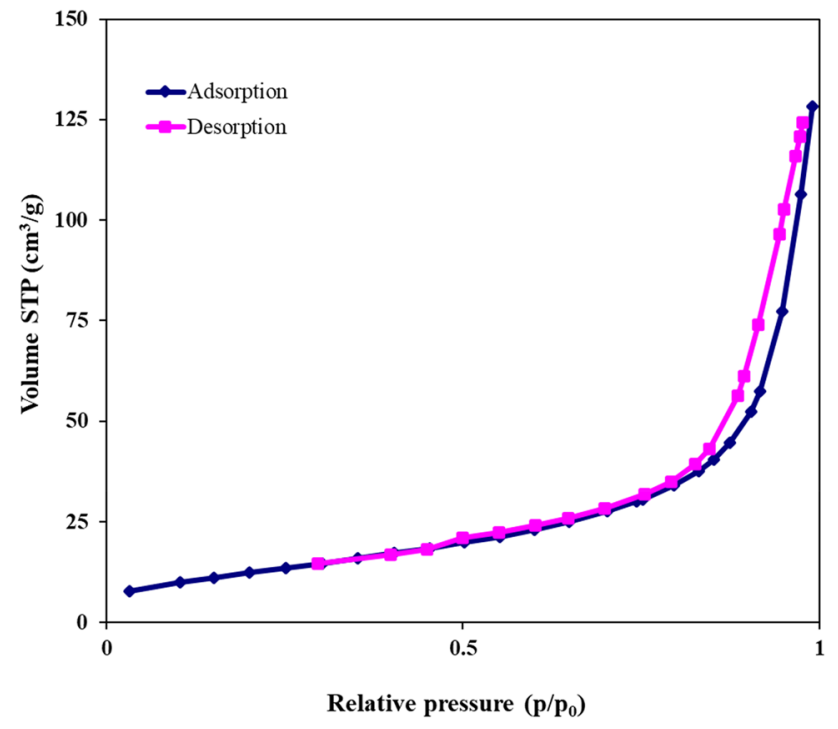

Figure 7. BET nitrogen adsorption/desorption isotherm of algal $\mathrm{NiO}$ nanocatalyst.

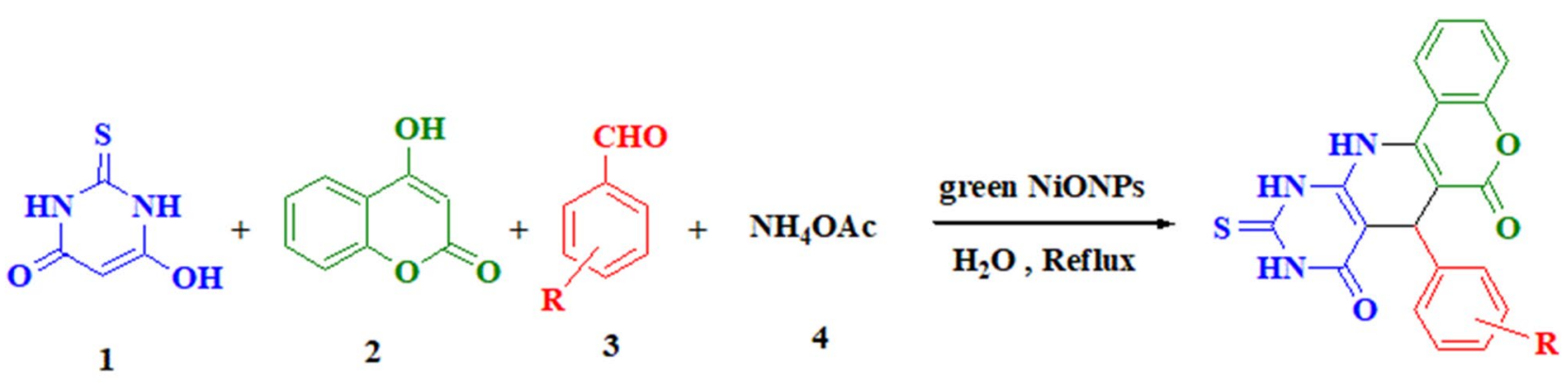

$5 \mathbf{a}-\mathbf{j}$

Scheme 2. Synthesis of pyridopyrimidine derivatives $\mathbf{4 a - j}$ catalyzed by biogenic NiO NPs via a four-component reaction.

\begin{tabular}{|l|l|l|l|}
\hline Entry & Catalytic conc. (mol\%) & Time (min) & Yield (\%) \\
\hline 1 & 2 & 40 & 75 \\
\hline 2 & 5 & 40 & 96 \\
\hline 3 & 10 & 40 & 95 \\
\hline 4 & 15 & 40 & 90 \\
\hline 5 & 20 & 40 & 85 \\
\hline
\end{tabular}

Table 2. Optimization of catalytic amount of green NiO NPs in the aqueous synthesis of $\mathbf{5 g}$.

diameter were $45.59 \mathrm{~m}^{2} \mathrm{~g}^{-1}, 17.39 \mathrm{~nm}, 10.47 \mathrm{~cm}^{3} \mathrm{~g}^{-1}$, respectively. As a result, the algae-mediated biosynthesized $\mathrm{NiO}$ NPs could deem as mesoporous material accelerating the catalyzed-organic synthesis reaction catalyzedorganic synthesis reactions ${ }^{59}$.

Catalytic performance of biological NiO NPs. In order to determine the optimal conditions of the reaction, we investigate the different amount of $\mathrm{NiO}$ catalyst required for the environmentally friendly synthesis of pyridopyrimidine derivatives using a straightforward four-component reaction approach of thiobarbituric acid 1, 4-hydroxy coumarin $\mathbf{2}$, aldehyde $\mathbf{3}$, and ammonium acetate $\mathbf{4}$ as model substrates in water solvent condition (Scheme 2). It is found that the best yield of products (95\%) was achieved when the molar concentration of nanoparticles reached 5\% $(0.004 \mathrm{mmol}, 3 \mathrm{mg})$ (Table 2). It is noted that neither yield nor rate was revealed significant improvement by loading a larger amount of $\mathrm{NiO}$ nanocatalyst as illustrated in Table 2 .

To assess the effectiveness of the catalytic activity of generated NiO NPs, a test reaction was explored in presence of various catalysts with respect to the reaction time of choice in an aqueous medium (Table 3). Generally, 


\begin{tabular}{|l|l|l|l|c|l|}
\hline Entry & Catalyst & Time (min) & TON & TOF & Yield (\%) \\
\hline 1 & Bulk Ni oxide & 240 & 12,200 & 50.83 & 61 \\
\hline 2 & Commercial NiO NPs & 60 & 16,000 & 266.66 & 80 \\
\hline 3 & Acetic acid & 120 & 10,000 & 83.33 & 50 \\
\hline 4 & Biogenic NiO NPs & 40 & 19,200 & 480.0 & 96 \\
\hline 5 & HCl & 90 & 6,000 & 66.67 & 30 \\
\hline 6 & Neat & Overnight & - & - & Trace \\
\hline
\end{tabular}

Table 3. Comparison the efficacy of different catalysts in $\mathbf{5 g}$ product preparation.
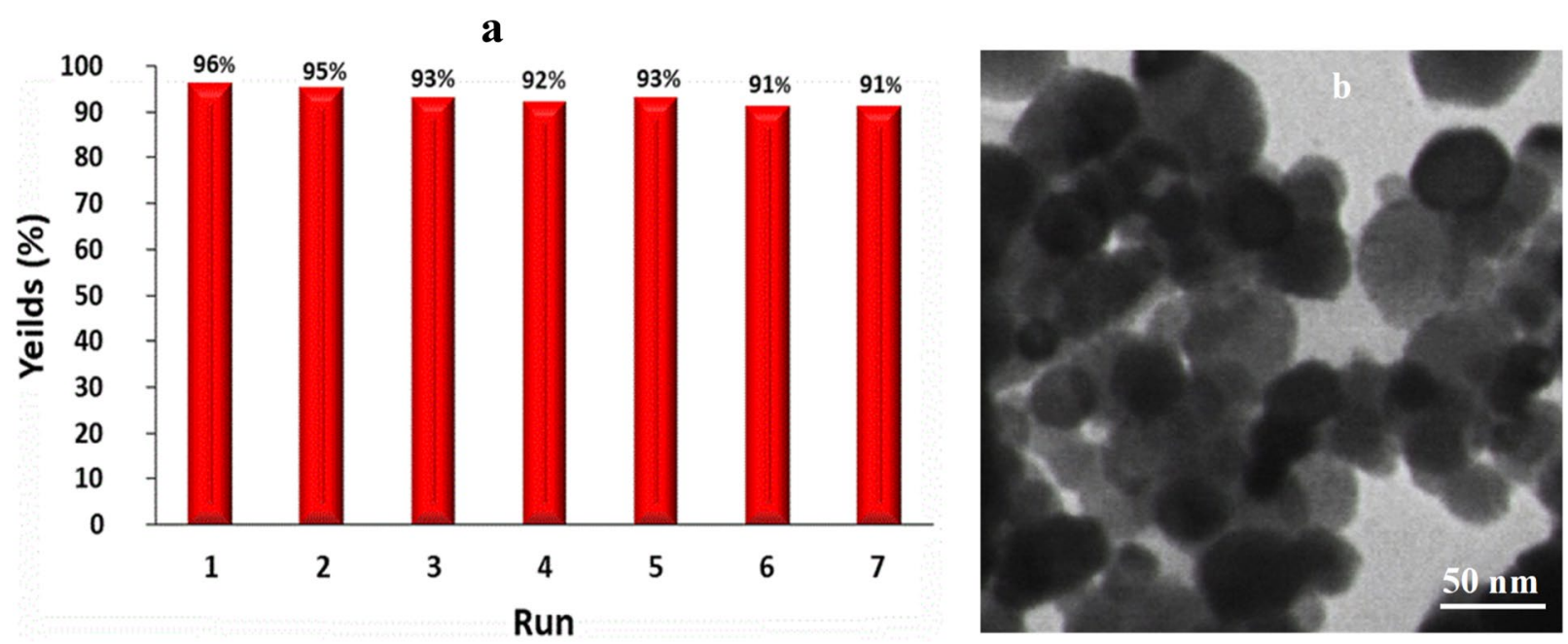

Figure 8. (a) Reusability and (b) TEM image of recovered algal NiO nanocatalyst in the synthesis of $\mathbf{5} \mathbf{g}$ in water.

in the absence of a catalyst at a consistent temperature of $40^{\circ} \mathrm{C}$ only offered trace yield of the expected products (entry 6). The results showed that the highest yield of products was obtained when biogenic NiO NPs contributed to the model reaction (entry 4). Based on TON (turnover number) and TOF (turnover frequency) results, the algal NiO NPs revealed greater stability and higher efficiency rather than other catalysts such as chemical bare NiO NPs (Table 3). Moreover, introducing chemical-produced NiO NPs (entry 2) increases the resultants yield rather than conventional catalysts (entries $1,3,5$ ) in a shorter time whereas it produced lower yield and a longer reaction time rather than algal NiO NPs (entry 4). It is found that the occurrence of plant-derived phytochemical constituents on the surface of bio-assisted nanoparticles during the synthesis process, would tune the distinctive parameters of decorated nanomaterials such as morphology and particle-size distribution which in turn improve their catalytic properties rather than plain traditional catalysts ${ }^{6}$. For instance, the utilized biogenic NiO NPs in this study exhibited shorter reaction times and higher yields of products in comparison to poly (4-styrenesulfonic acid) mesoporous graphene oxide hybrid (PSSA-MGO) catalyst in the synthetic reaction of alike pyridopyrimidines ${ }^{60}$. As a result, the designed algal NiO NPs-catalyzed organic synthesis takes major advantages including excellent yields, short time, low cost, simplicity, green chemical reaction condition, and environmental sustainability.

Recyclability of the nanocatalyst. The recycling of the catalyst is a pivotal point in the procedure of organic synthesis. In order to probe the reusability of biological magnetic NiO NPs; it was isolated from the reaction medium via an external magnet and washed several times with plenty of ethanol and water to achieve an unpolluted catalyst. It is found that no noticeable deterioration in the catalytic performance of algal NiO NPs was detected when it subjected to successive catalytic runs (up to seven) as depicted in Fig. 8a. The TEM image indicated that the morphology of the recovered nano-scaled $\mathrm{NiO}$ catalyst even after seven successive cycles relatively show no significant change after the reaction (Fig. 8b). In addition, the results of FTIR and EDX spectra exhibited no significant changes in surface chemistry and elemental composition indicating durability and stability reused biogenic $\mathrm{NiO}$ nanocatalyst (Fig. S2). Therefore, the desired pyridopyrimidine derivatives could furnish in the proficient yield in the presence of bioprepared NiO NPs.

Scope and limits of biogenic NiO NPs efficiency. The generality of catalytic efficiency of NiO NPs was investigated using a library of miscellaneous functionalized organic aldehydes moieties under the optimized reaction conditions. Regardless of induced substituents, the produced pyridopyrimidine derivatives furnished in high product yields with appreciable purity, confirming the profound effect of green NiO NPs. Nevertheless, the 


\begin{tabular}{|c|c|c|c|c|c|c|c|c|}
\hline Entrya & $\mathbf{R}$ & Product & Time (min) & TONc & TOFd (min-1) & Yieldb (\%) & MP ( $\left.{ }^{\circ} \mathrm{C}\right)$ (Ref.) & \\
\hline \multirow[t]{2}{*}{1} & $\mathrm{H}$ & & $5 a$ & 45 & 17,600 & 391 & 88 & $235-237$ \\
\hline & & & & & & & & $(236-238)^{60}$ \\
\hline \multirow[t]{2}{*}{2} & 2-Cl & & $5 b$ & 47 & 18,400 & 391.49 & 92 & $215-217$ \\
\hline & & & & & & & & $(218-220)^{60}$ \\
\hline \multirow[t]{2}{*}{3} & 3-Cl & & $5 c$ & 50 & 18,000 & 360 & 90 & $220-222$ \\
\hline & & & & & & & & $(219-220)^{60}$ \\
\hline \multirow[t]{2}{*}{4} & 4-Cl & & $5 \mathrm{~d}$ & 42 & 18,800 & 447.62 & 94 & $246-248$ \\
\hline & & & & & & & & $(244-246)^{60}$ \\
\hline Continu & & & & & & & & \\
\hline
\end{tabular}




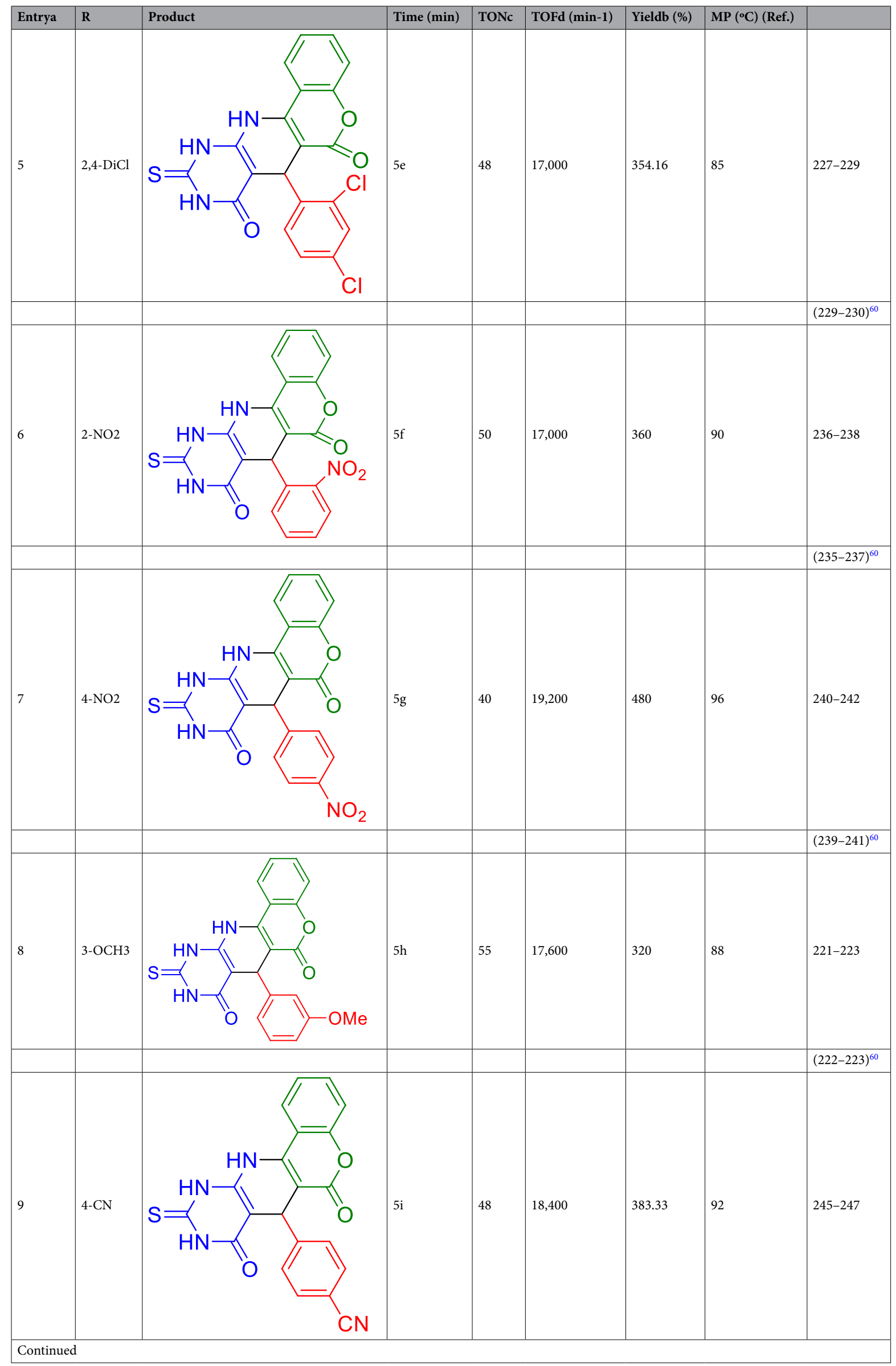




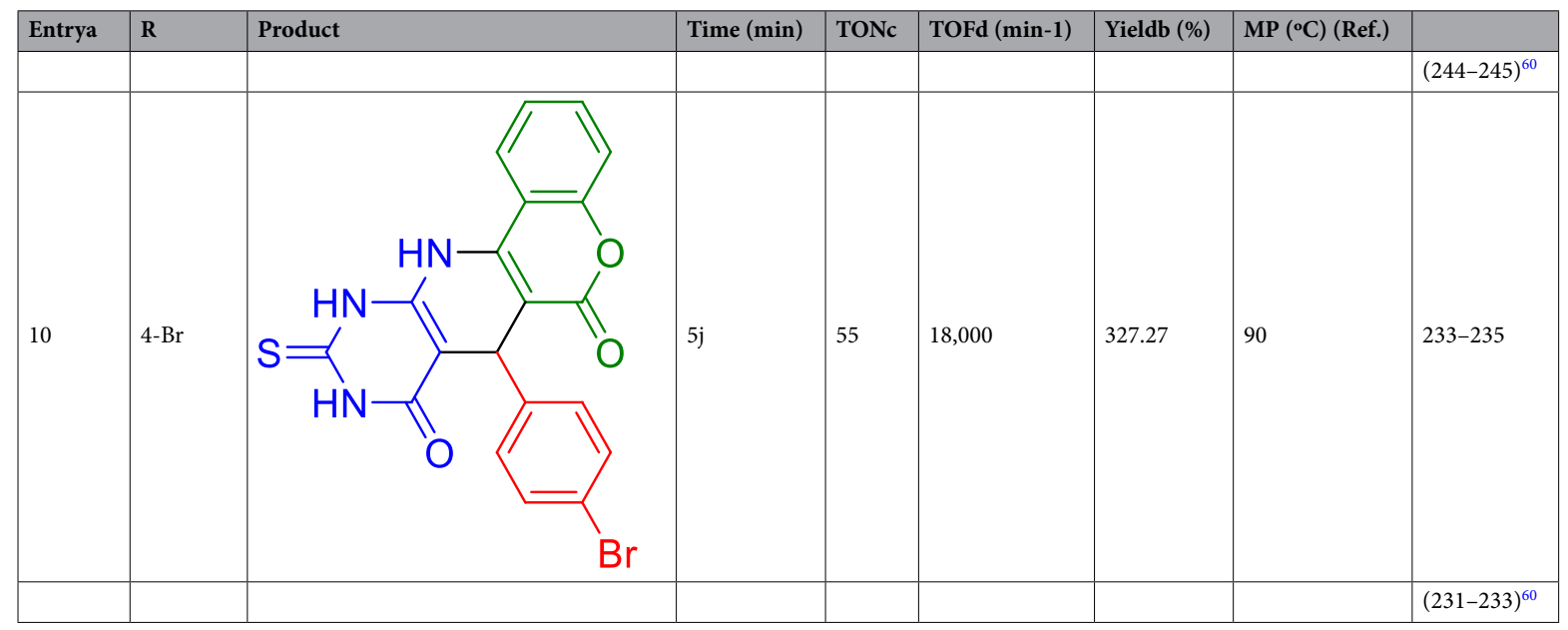

Table 4. NiO NPs-catalyzed the synthesis of pyridopyrimidine derivatives ${ }^{\mathrm{a}}$.<smiles>[R]c1ccc(C2c3c([nH]c(=S)[nH]c3=O)Nc3c2c(=O)oc2ccccc32)cc1</smiles>

${ }^{a}$ Reaction conditions: 4-hydroxy coumarin $(1 \mathrm{mmol})$, ammonium acetate $(1 \mathrm{mmol})$, thiobarbituric acid $(1 \mathrm{mmol})$, aromatic aldehyde $(1 \mathrm{mmol})$, and NiO NPs $(5 \% \mathrm{~mol}) .{ }^{\mathrm{b}}$ Isolated yield. ${ }^{\mathrm{c}} \mathrm{TON}=$ turnover number $=$ mol of product $/$ mole of catalyst. ${ }^{d} \mathrm{TOF}=$ turnover frequency $=$ mol of product $/$ mole of catalyst per $\min$.

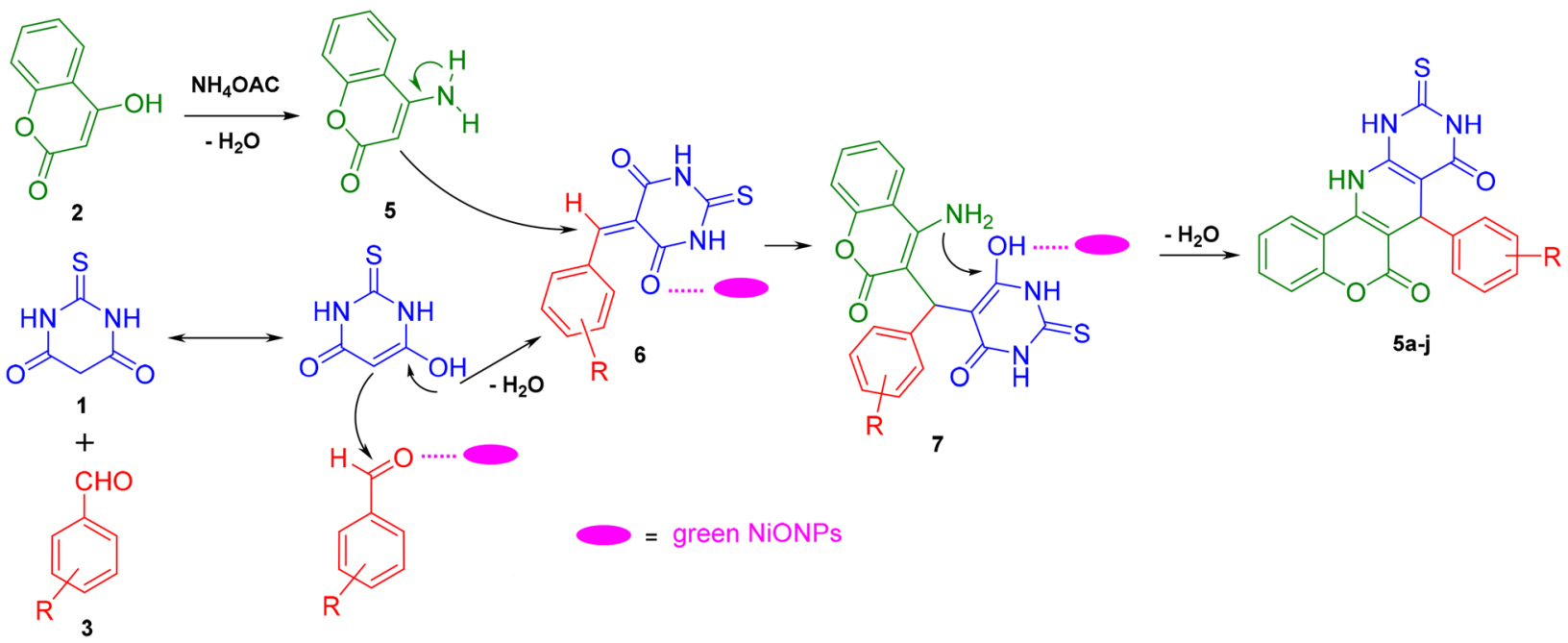

Scheme 3. A proposed mechanism for the fabrication of pyridopyrimidine derivatives on the surface of biogenic $\mathrm{NiO}$ nanocatalyst.

variation of electron-donating and electron-withdrawing groups relatively change the yield of products. Moreover, the highest increase in the rate and product yield was acquired when the $\mathrm{NO}_{2}$ group was replaced at the para-position of aryl aldehyde producing the target product (5g) with a yield of $96 \%$ (Table 4, entry 7, Fig. S1).

Pyridopyrimidines synthesis mechanism. According to the literature, a possible mechanism is proposed in Scheme 3 showing the pathway of catalyzed sequential multicomponent reactions. In the initial step, the enolic form of thiobarbituric acid (1) reacts with the catalyst-activated carbonyl of aryl aldehyde (3) through Knoevenagel condensation, to generate the $\alpha, \beta$-unsaturated compound 6 followed by a dehydration reaction. In the next step, a reaction between 4-hydroxy coumarin (2) and in situ produced ammonia from ammonium acetate (4) is proposed to give 4 -amino coumarin (5) at $100^{\circ} \mathrm{C}$. Then, via Michael addition of enamine (5) to an 
alpha, beta-unsaturated carbonyl acceptor of $\mathbf{6}$, the intermediate 7 is formed. In the final step, the intramolecular ring cyclization occurs with an amino group attack on the carbonyl group with aid of $\mathrm{NiO}$ catalyst after lossing of $\mathrm{H}_{2} \mathrm{O}$. As a result, the desired products $\mathbf{5 a - 1}$ are promoted within an aqueous medium at the appropriate time (Scheme 3). Apparently, introducing algal NiO NPs to the designed reaction would simultaneously boost the electrophilic character of the reactants and facilitate the attack of nucleophile groups owing to its acidic character. In addition, Lewis acid $\mathrm{NiO}$ nanocatalyst increases the generated intermediate stability and enhances the reactivity of organic materials as well ${ }^{61,62}$.

\section{Conclusion}

We have demonstrated an efficient synthetic approach for the biosynthesis of nickel oxide nanoparticles using marine red algae extract as a novel natural source in the absence of hazardous reagents. It is found that algaederived phytochemicals are effectively involved in bioreduction and coating as-prepared nanoparticles in benign conditions. The formation of NiO NPs was confirmed by using UV-vis, FTIR, XRD, TEM, TGA, BET, and EDX techniques. According to TON and TOF results the algal NiO NPs function as a highly robust catalyst for the one-pot facile preparation of pyridopyrimidine derivatives in water as a green solvent. The simple magnetic separation and high reusability of up to seven consecutive cycles further indicated the substantial catalytic activity of green NiO NPs. The sustainability, economic feasibility, and environmental compatibility of the proposed procedure could provide a broad range of uses in the processing and green synthesis of organic compounds.

Materials and methods. All utilized chemicals in this study were obtained from Merck and used as received without any further purification. The formation of biofabricated NiO NPs was scrutinized using a UVvis (Analytic Jena-Germany) spectrophotometer in the spectral range of $200-700 \mathrm{~nm}$. To detect content variations of the functional groups, the FTIR spectra of the materials were recorded on Nicolet MAGNA-IR 550 spectrometer (Madison, WI, USA) by a $\mathrm{KBr}$ pellet. X-ray powder diffraction (XRD) diffractometer (Cu Ka, radiation, $\lambda=1.5405 \AA$ ) was run at a scanning speed of $2 / \mathrm{min}$ from 10 to $80(2 \theta)$, examining the crystal structure of nanoparticles. To explore the magnetic properties of the nanoparticles, a vibrating sample magnetometer. (VSM, model BHV-55, Riken, Japan) the experiment was operated with a magnetic field up to $10 \mathrm{kOe}$. The morphology and particle size were obtained via transmission electron microscopy (TEM) operating with a Leo 912 $\mathrm{AB}$ at an accelerating voltage of $200 \mathrm{kV}$. For organic product characterization, melting points were gauged using the electrothermal IA9200 apparatus. Compositional analysis of the sample was carried out by X-ray energy dispersive spectroscopy (EDX). The progress of the reactions and assessment of the purity of the substrate was followed with TLC using silica gel SILG/UV 254 and 365 plates. To probe the surface of the sample, thermogravimetric analysis (TGA, PerkinElmer, Pyris 1, USA) technique was used. The surface area per mass of nanosample was measured using Brunauer-Emmett-Teller (BET) method. Finally, the specimens of red marine algae were gathered from the intertidal zone in coastal areas of Bushehr province, Iran, during low tide.

Preparation of marine algae extract. In order to obtain a proper extract, in a $100 \mathrm{ml}$ beaker $2 \mathrm{~g}$ of washed and under shade-dried powdered algae was soaked in $20 \mathrm{ml}$ of distilled water under stirring at $180 \mathrm{rpm}$ using a magnetic stirrer for $1 \mathrm{~h}$ then sonicated at $70{ }^{\circ} \mathrm{C}$ for $25 \mathrm{~min}$. Further, the mixture was boiled for $10 \mathrm{~min}$ then cooled to room temperature and filtered through Whatman filter paper Grade No 1 (pore size: $11 \mu \mathrm{m}$ ). Eventually, the resultant crude extract was stored in an air-tight container and placed in the refrigerator for additional processing.

Phyto-synthesis of NiO NPs. To synthesize nickel oxide nanoparticles, $10 \mathrm{ml}$ of an aqueous solution of $\mathrm{NiCl}_{2} \cdot 6 \mathrm{H}_{2} \mathrm{O}(0.01 \mathrm{M})$ was added to $90 \mathrm{ml}$ of marine red algae extract. The reaction mixture containing red algae extract and nickel chloride hydrate solution was magnetically stirred at $800 \mathrm{rpm}$ for $30 \mathrm{~min}$ at $60^{\circ} \mathrm{C}$ to attain a homogeneous solution. After cooling down to room temperature, the purification of the resulting solution was fulfilled through continuous centrifugation at $12,000 \mathrm{rpm}$ for $20 \mathrm{~min}$ with distilled water and ethanol to eliminate undesirable impurities followed by decanting. The obtained precipitate specimen was annealed at $450{ }^{\circ} \mathrm{C}$ for $90 \mathrm{~min}$.

Synthesis of pyridopyrimidines derivatives over NiO NPs catalyst. A mixture of ammonium acetate $(4,1 \mathrm{mmol})$ and 4 -hydroxy coumarin $(2,1 \mathrm{mmol})$ was refluxed in water $(15 \mathrm{ml})$ for $30 \mathrm{~min}$. Then, thiobarbituric acid $(\mathbf{1}, 1 \mathrm{mmol})$, and aromatic aldehyde $(3,1 \mathrm{mmol})$ in the presence of green $\mathrm{NiO} \mathrm{NPs}(5 \mathrm{~mol} \%, 0.003 \mathrm{~g})$ as a heterogeneous catalyst were added and the reaction was stirred for further $10 \mathrm{~min}$ (Scheme 2). Upon completion of the reaction as monitored by TLC, the reaction solution was allowed to cool down to room temperature, subsequently, the $50 \mathrm{H}_{2} \mathrm{O}$ was added to precipitate, filtered out and dried. The solid magnetic nanocatalyst was then separated by centrifugation and an external magnetic force, washed with $10 \mathrm{ml}$ of pure ethanol, dried at $80^{\circ} \mathrm{C}$ overnight, and reused. In order to afford uncontaminated products, the precipitate was boiled in $15 \mathrm{~mL}$ of $\mathrm{EtOH}$ for $5 \mathrm{~min}$, and the final crystalline products were characterized using FTIR, elemental analysis, ${ }^{1} \mathrm{H}$ and ${ }^{13} \mathrm{C}$ NMR spectroscopic techniques (Figs. S1, see supplementary).

Received: 21 November 2020; Accepted: 8 March 2021

Published online: 18 March 2021 


\section{References}

1. Patil, M. P. \& Kim, G.-D. Marine microorganisms for synthesis of metallic nanoparticles and their biomedical applications. Colloids Surf. B 172, 487-495 (2018).

2. Bajpai, V. K. et al. Developments of cyanobacteria for nano-marine drugs: relevance of nanoformulations in cancer therapies. Mar. Drugs 16, 179 (2018).

3. Khalafi, T., Buazar, F. \& Ghanemi, K. Phycosynthesis and enhanced photocatalytic activity of zinc oxide nanoparticles toward organosulfur pollutants. Sci. Rep. 9, 1-10 (2019).

4. Mohanta, D. \& Ahmaruzzaman, M. Addressing nanotoxicity: green nanotechnology for a sustainable future. The ELSI Handbook of Nanotechnology: Risk, Safety, ELSI and Commercialization, 103-112 (2020).

5. Koopi, H. \& Buazar, F. A novel one-pot biosynthesis of pure alpha aluminum oxide nanoparticles using the macroalgae Sargassum ilicifolium: a green marine approach. Ceram. Int. 44, 8940-8945 (2018).

6. Sepahvand, M., Buazar, F. \& Sayahi, M. H. Novel marine-based gold nanocatalyst in solvent-free synthesis of polyhydroquinoline derivatives: Green and sustainable protocol. Appl. Organomet. Chem. 34, 1-11 (2020).

7. LewisOscar, F. et al. Algal nanoparticles: synthesis and biotechnological potentials. Algae-Organ Imminent Biotechnol 7, 157-182 (2016).

8. Kalidindi, S. B. \& Jagirdar, B. R. Nanocatalysis and prospects of green chemistry. Chemsuschem 5, 65-75 (2012).

9. Polshettiwar, V. \& Varma, R. S. Green chemistry by nano-catalysis. Green Chem. 12, 743-754 (2010).

10. Zeng, H. C. Integrated nanocatalysts. Acc. Chem. Res. 46, 226-235 (2013).

11. Ghorbani-Choghamarani, A., Seydyosefi, Z. \& Tahmasbi, B. Tribromide ion supported on boehmite nanoparticles as a reusable catalyst for organic reactions. C. R. Chim. 21, 1011-1022 (2018).

12. Nikoorazm, M., Tahmasbi, B., Gholami, S. \& Moradi, P. Copper and nickel immobilized on cytosine@ MCM-41: as highly efficient, reusable and organic-inorganic hybrid nanocatalysts for the homoselective synthesis of tetrazoles and pyranopyrazoles. Appl. Organ. Chem. 34, e5919 (2020).

13. Ghorbani-Choghamarani, A., Moradi, P. \& Tahmasbi, B. Modification of boehmite nanoparticles with Adenine for the immobilization of $\mathrm{Cu}$ (II) as organic-inorganic hybrid nanocatalyst in organic reactions. Polyhedron 163, 98-107 (2019).

14. Nikoorazm, M., Rezaei, Z. \& Tahmasbi, B. Two Schiff-base complexes of copper and zirconium oxide supported on mesoporous MCM-41 as an organic-inorganic hybrid catalysts in the chemo and homoselective oxidation of sulfides and synthesis of tetrazoles. J. Porous Mater. 27, 671-689 (2020).

15. Maleki, A., Rahimi, J., Demchuk, O. M., Wilczewska, A. Z. \& Jasiński, R. Green in water sonochemical synthesis of tetrazolopyrimidine derivatives by a novel core-shell magnetic nanostructure catalyst. Ultrason. Sonochem. 43, 262-271 (2018).

16. Sachdeva, H., Dwivedi, D., Bhattacharjee, R., Khaturia, S. \& Saroj, R. NiO nanoparticles: an efficient catalyst for the multicomponent one-pot synthesis of novel spiro and condensed indole derivatives. J. Chem. 2013, 1-10 (2013).

17. Kaur, N. Nickel catalysis: six membered heterocycle syntheses. Synth. Commun. 49, 1103-1133 (2019).

18. Halder, M. et al. Sustainable generation of Ni (OH) 2 nanoparticles for the green synthesis of 5-substituted $1 \mathrm{H}$-tetrazoles: a competent turn on fluorescence sensing of H2O2. ACS Omega 3, 8169-8180 (2018).

19. Reddy, B. P., Iniyavan, P., Sarveswari, S. \& Vijayakumar, V. Nickel oxide nanoparticles catalyzed synthesis of poly-substituted quinolines via Friedlander hetero-annulation reaction. Chin. Chem. Lett. 25, 1595-1600 (2014).

20. Nasseri, M. A., Kamali, F. \& Zakerinasab, B. Catalytic activity of reusable nickel oxide nanoparticles in the synthesis of spirooxindoles. RSC Adv. 5, 26517-26520 (2015).

21. Ghorbani-Choghamarani, A., Moradi, P. \& Tahmasbi, B. Nickel (II) immobilized on dithizone-boehmite nanoparticles: as a highly efficient and recyclable nanocatalyst for the synthesis of polyhydroquinolines and sulfoxidation reaction. J. Iran. Chem. Soc. 16, 511-521 (2019).

22. Ahghari, M. R., Soltaninejad, V. \& Maleki, A. Synthesis of nickel nanoparticles by a green and convenient method as a magnetic mirror with antibacterial activities. Sci. Rep. 10, 1-10 (2020).

23. Imran Din, M. \& Rani, A. Recent advances in the synthesis and stabilization of nickel and nickel oxide nanoparticles: a green adeptness. Int. J. Anal. Chem. 2016 (2016).

24. Iqbal, J. et al. Phytogenic synthesis of nickel oxide nanoparticles (NiO) using fresh leaves extract of Rhamnus triquetra (wall.) and investigation of its multiple in vitro biological potentials. Biomedicines 8, 117 (2020).

25. Haider, A. et al. Green synthesized phytochemically (Zingiber officinale and Allium sativum) reduced nickel oxide nanoparticles confirmed bactericidal and catalytic potential. Nanoscale Res. Lett. 15, 1-11 (2020).

26. Maleki, A. \& Firouzi-Haji, R. 1-Proline functionalized magnetic nanoparticles: a novel magnetically reusable nanocatalyst for one-pot synthesis of 2, 4, 6-triarylpyridines. Scientific reports 8, 1-8 (2018).

27. Maleki, A. One-pot multicomponent synthesis of diazepine derivatives using terminal alkynes in the presence of silica-supported superparamagnetic iron oxide nanoparticles. Tetrahedron Lett. 54, 2055-2059 (2013).

28. Ghorbani-Choghamarani, A. et al. Nano aluminium nitride as a solid source of ammonia for the preparation of Hantzsch 1 , 4-dihydropyridines and bis-(1, 4-dihydropyridines) in water via one pot multicomponent reaction. J. Braz. Chem. Soc. 22, 525-531 (2011).

29. Atashkar, B., Rostami, A., Gholami, H. \& Tahmasbi, B. Magnetic nanoparticles Fe 3 O 4-supported guanidine as an efficient nanocatalyst for the synthesis of $2 \mathrm{H}$-indazolo $[2,1-\mathrm{b}]$ phthalazine-triones under solvent-free conditions. Res. Chem. Intermed. 41, 3675-3681 (2015).

30. Maleki, A. One-pot three-component synthesis of pyrido [2', 1': 2, 3] imidazo [4, 5-c] isoquinolines using Fe 3 O $4 @$ SiO 2-OSO $3 \mathrm{H}$ as an efficient heterogeneous nanocatalyst. RSC Adv. 4, 64169-64173 (2014).

31. Maleki, A., Hajizadeh, Z. \& Salehi, P. Mesoporous halloysite nanotubes modified by CuFe 2 O 4 spinel ferrite nanoparticles and study of its application as a novel and efficient heterogeneous catalyst in the synthesis of pyrazolopyridine derivatives. Sci. Rep. 9, $1-8$ (2019).

32. Maleki, A. Fe3O4/SiO2 nanoparticles: an efficient and magnetically recoverable nanocatalyst for the one-pot multicomponent synthesis of diazepines. Tetrahedron 68, 7827-7833 (2012).

33. Maleki, A. \& Aghaei, M. Ultrasonic assisted synergetic green synthesis of polycyclic imidazo (thiazolo) pyrimidines by using Fe3O4@ clay core-shell. Ultrason. Sonochem. 38,585-589 (2017).

34. Maleki, A. \& Aghaei, M. Sonochemical rate enhanced by a new nanomagnetic embedded core/shell nanoparticles and catalytic performance in the multicomponent synthesis of pyridoimidazoisoquinolines. Ultrason. Sonochem. 38, 115-119 (2017).

35. DeGoey, D. A. et al. Discovery of pyrido [2, 3-d] pyrimidine-based inhibitors of HCV NS5A. Bioorg. Med. Chem. Lett. 23, 3627-3630 (2013).

36. Nasr, M. N. \& Gineinah, M. M. Pyrido [2, 3-d] pyrimidines and Pyrimido $\left[5^{\prime}, 4^{\prime}: 5,6\right]$ pyrido $[2,3$-d] pyrimidines as new antiviral agents: synthesis and biological activity. Archiv der Pharmazie Int. J. Pharmaceut. Med. Chem. 335, $289-295$ (2002).

37. Tandel, H. T. \& Patel, S. K. Efficient procedure with new fused pyrido [2,3-d] pyrimidine derivatives as potent antimicrobial agents. (2020).

38. Colbry, N. L., Elslager, E. F. \& Werbel, L. M. Folate antagonists. 21. Synthesis and antimalarial properties of 2, 4-diamino-6(benzylamino) pyrido [3, 2-d] pyrimidines. J. Med. Chem. 28, 248-252 (1985). 
39. Mohire, P. P. et al. An expedient four component synthesis of substituted pyrido-pyrimidine heterocycles in glycerol: proline based low transition temperature mixture and their antioxidant activity with molecular docking studies. Polycyclic Arom. Compound., $1-19(2020)$.

40. D’agostino, L. A. et al. Substituted pyrido [2, 3-d] pyrimidines as inhibitors of protein kinases. USA patent (2020).

41. Kots, A. Y. et al. Pyridopyrimidine derivatives as inhibitors of cyclic nucleotide synthesis: application for treatment of diarrhea. Proc. Natl. Acad. Sci. 105, 8440-8445 (2008).

42. Dasari, S. R., Tondepu, S., Vadali, L. R. \& Seelam, N. PEG-400 mediated an efficient eco-friendly synthesis of new isoxazolyl pyrido [2, 3-d] pyrimidines and their anti-inflammatory and analgesic activity. Synth. Commun., 1-12 (2020).

43. Shamroukh, A., Rashad, A. \& Abdelmegeid, F. The chemistry of pyrido [2, 3-d] pyrimidines and their applications. J. Chem. Pharm. Res. 8, 734-772 (2016).

44. Rezazadeh, N. H., Buazar, F. \& Matroodi, S. Synergistic effects of combinatorial chitosan and polyphenol biomolecules on enhanced antibacterial activity of biofunctionalaized silver nanoparticles. Sci. Rep. 10, 1-13 (2020).

45. Romney, D. K., Arnold, F. H., Lipshutz, B. H. \& Li, C.-J. Chemistry takes a bath: reactions in aqueous media. J. Org. Chem. 83, 7319-7322 (2018).

46. Olajire, A. \& Mohammed, A. Green synthesis of nickel oxide nanoparticles and studies of their photocatalytic activity in degradation of polyethylene films. Adv. Powder Technol. 31, 211-218 (2020).

47. El-Kemary, M., Nagy, N. \& El-Mehasseb, I. Nickel oxide nanoparticles: synthesis and spectral studies of interactions with glucose. Mater. Sci. Semicond. Process. 16, 1747-1752 (2013).

48. Srihasam, S., Thyagarajan, K., Korivi, M., Lebaka, V. R. \& Mallem, S. P. R. Phytogenic generation of NiO nanoparticles using Stevia leaf extract and evaluation of their in-vitro antioxidant and antimicrobial properties. Biomolecules 10, 89 (2020).

49. Diallo, A. et al. Structural, optical and photocatalytic applications of biosynthesized NiO nanocrystals. Green Chem. Lett. Rev. 11, $166-175$ (2018).

50. Ferreira, T. \& Rasband, W. ImageJ user guide. ImageJ/Fiji 1, 155-161 (2012).

51. Buazar, F., Sweidi, S., Badri, M. \& Kroushawi, F. Biofabrication of highly pure copper oxide nanoparticles using wheat seed extract and their catalytic activity: A mechanistic approach. Green Proc. Synth. 8, 691-702 (2019).

52. Balan, V. et al. Vibrational spectroscopy fingerprinting in medicine: from molecular to clinical practice. Materials 12, 2884 (2019).

53. Moradi, P., Hajjami, M. \& Tahmasbi, B. Fabricated copper catalyst on biochar nanoparticles for the synthesis of tetrazoles as antimicrobial agents. Polyhedron 175, 114169 (2020).

54. Dashtban, M., Kepka, G., Seiboth, B. \& Qin, W. Xylitol production by genetically engineered Trichoderma reesei strains using barley straw as feedstock. Appl. Biochem. Biotechnol. 169, 554-569 (2013).

55. Badrinathan, S., Shiju, T., Christa, A. S. S., Arya, R. \& Pragasam, V. Purification and structural characterization of sulfated polysaccharide from Sargassum myriocystum and its efficacy in scavenging free radicals. Indian J. Pharmaceut. Sci. 74, 549 (2012).

56. Sabouri, Z. et al. Plant-based synthesis of $\mathrm{NiO}$ nanoparticles using salvia macrosiphon Boiss extract and examination of their water treatment. Rare Metals, 1-11 (2019).

57. Madhu, G., Maniammal, K. \& Biju, V. Defect induced ferromagnetic interaction in nanostructured nickel oxide with core-shell magnetic structure: the role of Ni 2+ and O 2-vacancies. Phys. Chem. Chem. Phys. 18, 12135-12148 (2016).

58. Kalaie, M. R., Youzbashi, A. A., Meshkot, M. A. \& Hosseini-Nasab, F. Preparation and characterization of superparamagnetic nickel oxide particles by chemical route. Appl. Nanosci. 6, 789-795 (2016).

59. Barzinjy, A. A., Hamad, S. M., Aydın, S., Ahmed, M. H. \& Hussain, F. H. Green and eco-friendly synthesis of Nickel oxide nanoparticles and its photocatalytic activity for methyl orange degradation. J. Mater. Sci.: Mater. Electron. 31, 11303-11316 (2020).

60. Sayahi, M. H., Bahadorikhalili, S., Saghanezhad, S. J., Miller, M. A. \& Mahdavi, M. Sulfonic acid-functionalized poly (4-styrenesulfonic acid) mesoporous graphene oxide hybrid for one-pot preparation of coumarin-based pyrido [2, 3-d] pyrimidine-dione derivatives. Res. Chem. Intermed. 46, 491-507 (2020).

61. Kobayashi, S. \& Manabe, K. Green Lewis acid catalysis in organic synthesis. Pure Appl. Chem. 72, 1373-1380 (2000).

62. Safaei-Ghomi, J. \& Paymard-Samani, S. Facile and rapid synthesis of 5-substituted 1 H-tetrazoles via a multicomponent domino reaction using nickel (II) oxide nanoparticles as catalyst. Chem. Heterocycl. Compd. 50, 1567-1574 (2015).

\section{Author contributions}

J.M. conducted experiments associated with nickel oxide biosynthesis and their spectroscopic characterization. F.B. supervised the study, designed the research framework, and carried out the data analysis. M.H.S. participated in setting up an experiment of synthesis of pyridopyrimidines derivatives over NiO NPs catalyst and analyzing results. The final manuscript has been written and edited by F.Buazar with contributions from all coauthors.

\section{Competing interests}

The authors declare no competing interests.

\section{Additional information}

Supplementary Information The online version contains supplementary material available at https://doi.org/ 10.1038/s41598-021-85832-z.

Correspondence and requests for materials should be addressed to F.B.

Reprints and permissions information is available at www.nature.com/reprints.

Publisher's note Springer Nature remains neutral with regard to jurisdictional claims in published maps and institutional affiliations.

Open Access This article is licensed under a Creative Commons Attribution 4.0 International License, which permits use, sharing, adaptation, distribution and reproduction in any medium or format, as long as you give appropriate credit to the original author(s) and the source, provide a link to the Creative Commons licence, and indicate if changes were made. The images or other third party material in this article are included in the article's Creative Commons licence, unless indicated otherwise in a credit line to the material. If material is not included in the article's Creative Commons licence and your intended use is not permitted by statutory regulation or exceeds the permitted use, you will need to obtain permission directly from the copyright holder. To view a copy of this licence, visit http://creativecommons.org/licenses/by/4.0/.

(C) The Author(s) 2021 